ISSN 0258-7122

Bangladesh J. Agril. Res. 33(3) : 353-362, September 2008

\title{
EFFECTS OF WATER STRESS AT VARIOUS GROWTH STAGES ON THE PHYSIO-MORPHOLOGICAL CHARACTERS AND YIELD IN CHILLI
}

\author{
M. A. I. KHAN ${ }^{1}$, A. M. FAROOQUE ${ }^{2}$, M. A. $\mathrm{HAQUE}^{3}$ \\ M. A. RAHIM ${ }^{4}$ AND M. A. HOQUE ${ }^{5}$
}

\begin{abstract}
An experiment was carried out during October 1999 to January 2000 in the field of Horticulture Department of Bangladesh Agricultural University, Mymensingh with two chilli accessions viz, C-0277 and C-0272 of moisture stress tolerant and susceptible type. The chilli accessions were put into trial under field condition to observe the effects of different water treatments imposed at their vegetative, flowering, and fruiting stages of growth and development. Results of the experiment revealed that accession C-0277 was water stress tolerant compared to accession C-0272. Watering at 4-day interval significantly gave the highest yield and dry matter. While, watering imposed in fruiting stage produced the maximum yield and dry matter in chilli.
\end{abstract}

Key Words: Chilli accessions, field condition, water stress, yield.

\section{Introduction}

Chilli is an important cash crop of Bangladesh. Two cultivated species-Capsicum annuum and Capsicum fruitescens are grown largely under rainfed condition throughout Bangladesh. The chilli farmers of Bangladesh cultivate local cultivars which produce very low yields. The main reasons of low yield are lacking of high yielding varieties and unavailability of irrigation facilities. Two accessions of chilli, moisture stress tolerant and susceptible types were identified through previous research works. The accessions were identified as such under glasshouse condition. But to establish as a moisture stress tolerant and susceptible variety, they must perform in the same manner under field condition. Establishment of moisture stressw tolerant and susceptible varieties have got high economic and commercial values in chilli cultivation of Bangladesh. In order to achieve ultimate goal of establishing tolerant and susceptible chilli variety, the previously selected two chilli accessions must put into trial in the field to know the effects of moisture stress on their yield and physio-morphological characters. With this view, the present experiment was undertaken.

${ }^{1}$ Chief Scientific Officer, Regional Agricultural Research Station, BARI, Akbarpur, Moulvibazar, ${ }^{2}$ Vice Chancellor, Sher-e-Bangla Agriculture University, Dhaka, ${ }^{3}$ Professor, Dept. of Crop Botany, Bangladesh Agricultural University, Mymensingh and ${ }^{4}$ Professor, Dept. of Horticulture, Bangladesh Agricultural University, Mymensingh, ${ }^{5}$ Senior Scientific Officer, Tuber Crops Research Sub Centre, BARI, Munshiganj, Bnangladesh. 


\section{Materials and Method}

The experiment was conducted at the field laboratory of Horticulture Department of Bangladesh Agricultural University (BAU), Mymensingh during October 1999 to January 2000. Two chilli accessions viz, C-0277 and C-0272 identified as water stress tolerant and susceptible types were selected as experimental materials. The accessions were originally collected from the stock of Spices Research Centre of Bangladesh Agricultural Research Institute, Joydebpur, Gazipur. The field was prepared by ploughing and cross ploughing followed by laddering. The bigger clods were crushed and made a fine tilth for lay out of the experiment. The seedlings were raised in $1.5 \mathrm{~m} \times 1.0 \mathrm{~m}$ size tray and twenty-day old seedlings were transplanted in the field plots on 07 November 1999. Harvesting was done from 21 February 2000 to 18 March 2000. The field capacity of moisture was determined by using moisture meter in the experimental plots. The unit plot size was $1.2 \mathrm{~m} \times 1.2 \mathrm{~m}$ being $40 \mathrm{~cm} \times 40 \mathrm{~cm}$ planting distance with nine plants per plot. All the experimental plots and plant root zones were encircled with polythene by entering it $40 \mathrm{~cm}$ inside the soil so that no moisture could enter from outside the plot and root zones of the plants. The water treatments were imposed on 15 November1999. Split-split plot design was followed with 3 replications. Data were collected from 5 randomly selected plants in a plot. The collected data were analyzed statistically with MSTATC programme and means were separated by LSD test.

Water treatment was applied at 3 stages of growth and development viz., Vegetative stage, $S_{1}=28-43$ days, Flowering stage, $S_{2}=44-59$ days and Fruiting stage, $S_{3}=60-76$ days. Six water treatments were imposed $-W_{1}=$ watering once everyday, $\mathrm{W}_{2}=$ watering twice everyday, $\mathrm{W}_{3}=$ watering at 4 days interval, $\mathrm{W}_{4}=$ watering at 8 days interval, $\mathrm{W}_{5}=$ watering at 16 days interval and $\mathrm{W}_{0}=$ no watering (control). Irrigation treatments were applied starting from 15 November 1999 according to the treatment schedule mentioned above. Before application of water, moisture condition of the polythene encircled individual plant root zone was measured by the moisture meter. Only the deficit amount of moisture was added to the root zone to attain field capacity. Before starting addition of water, moisture meter was fixed to the soil of the polyethylene encircled root zone. Data recorded were number of leaves per plant, leaf area per plant, plant height, canopy diameter, root length, root volume, number of fruits per plant, fruit length, fruit diameter, individual fruit weight, fruit yield per plant, leaf dry weight, stem dry weight, root dry weight, and fruit dry weight. 


\section{Results and Discussion}

The single effect of the three factors, chilli accessions, growth stages, and water levels are shown in the Table 1, 2, and 3, resectively. The single effects of two chilli cultivars (accessions) on the physio-morphological characters are presented in Table 1. It is evident that the accession $V_{1}$ produced higher values for all the 15 parameters studied over the other accessions. The value of all the individual parameter differed significantly between the two accessions.

Table 1. Effect of two chili cultivars (accessions) on the physiomorphological characters

\begin{tabular}{l|l|l|l|c|c|c|c}
\hline Cultivar & $\begin{array}{c}\text { No. of } \\
\text { leaves/plant }\end{array}$ & $\begin{array}{c}\text { Leaf area/ } \\
\text { plant }\left(\mathrm{cm}^{2}\right)\end{array}$ & $\begin{array}{c}\text { Plant height } \\
(\mathrm{cm})\end{array}$ & $\begin{array}{c}\text { Canopy } \\
\text { diameter } \\
(\mathrm{cm})\end{array}$ & $\begin{array}{c}\text { Root length } \\
(\mathrm{cm})\end{array}$ & $\begin{array}{c}\text { Root } \\
\text { volume (cc) }\end{array}$ & $\begin{array}{c}\text { No. of } \\
\text { truits/plant }\end{array}$ \\
\hline $\mathrm{V}_{1}$ & 393.31 & 2163.44 & 74.39 & 52.89 & 528,34 & 11.27 & 189.24 \\
$\mathrm{~V}_{2}$ & 321.42 & 1721.90 & 64.24 & 37.21 & 423,31 & 9.24 & 137.91 \\
\hline $\mathrm{LSD}(0.05)$ & 9.90 & 32.72 & 2.38 & 1.511 & 23.64 & 0.341 & 8.44 \\
$\mathrm{CV}(\%)$ & 7.35 & 4.47 & 9.10 & 8.89 & 13.17 & 8,83 & 13.68 \\
\hline
\end{tabular}

Table 1. Cont'd.

\begin{tabular}{l|c|c|c|c|c|c|c|c}
\hline Cuitivar & $\begin{array}{c}\text { Fruit } \\
\text { length } \\
(\mathrm{mm})\end{array}$ & $\begin{array}{c}\text { Fruit } \\
\text { diameter } \\
(\mathrm{mm})\end{array}$ & $\begin{array}{c}\text { Individual } \\
\text { fruit wt (mg) }\end{array}$ & $\begin{array}{c}\text { Fruit } \\
\text { yield/ } \\
\text { plant (g) }\end{array}$ & $\begin{array}{c}\text { Leaf dry } \\
\text { wt (g) }\end{array}$ & $\begin{array}{c}\text { Stem dry } \\
\text { wt (g) }\end{array}$ & $\begin{array}{c}\text { Root dry } \\
\text { wt (g) }\end{array}$ & $\begin{array}{c}\text { Fruit dry } \\
\text { wt/ plant } \\
\text { (g) }\end{array}$ \\
\hline $\mathrm{V}_{1}$ & 34.40 & 8.17 & 476.32 & 89.67 & 4.40 & 9.15 & 3.26 & 18.28 \\
$\mathrm{~V}_{2}$ & 26.05 & 6.87 & 437.30 & 60.60 & 3.13 & 7.60 & 1.97 & 13.24 \\
\hline $\mathrm{I}, \mathrm{SD}(0.05)$ & 1,345 & 0.307 & 17.25 & 1.884 & 0.141 & 0.239 & 0.117 & 0.671 \\
$\mathrm{CV}(\%)$ & 11.80 & 10.81 & 13.32 & 12.98 & 9.90 & 7.56 & 11.78 & 11.29 \\
\hline
\end{tabular}

$* \mathrm{~V}_{1}=\mathrm{C} 0277$ and $\mathrm{V}_{2}=\mathrm{C} 0272$.

Single effect of growth stage is shown in Table 2. The table shows that the vegetative stage $S_{1}$ produced the highest value regarding individual fruit weight (549.6mg) only. The flowering stage $S_{2}$ produced the highest values for no. of leaves per plant (398.15), leaf area per plant $\left(2036.28 \mathrm{~cm}^{2}\right)$, plant height $(73.58 \mathrm{~cm})$, canopy diameter $(48.58 \mathrm{~cm})$, no. of fruits per plant (185.98), leaf dry weight $(4.02 \mathrm{~g})$, and stem dry weight $(9.02 \mathrm{~g})$. The fruiting stage $\mathrm{S}_{3}$ gave the highest values in case of root length $(523.62 \mathrm{~cm})$, root volume $(11.0 \mathrm{cc})$, fruit length (30.86mm), fruit yield per plan (79.40g), root dry veight (2.84g), and fruit dry weight $(17.74 \mathrm{~g})$. The values produced by the individual growth stage differed significantly among the 3 growth stages. 
Table 2. Effect of growth stages on the physio-morphological characters of two cultivars (accessions) of chilli.

\begin{tabular}{l|l|l|l|c|c|c|c}
\hline Growth & $\begin{array}{c}\text { No. of } \\
\text { leaves/plant }\end{array}$ & $\begin{array}{c}\text { Leaf area/ } \\
\text { plant }\left(\mathrm{cm}^{2}\right)\end{array}$ & $\begin{array}{c}\text { Plant } \\
\text { height }(\mathrm{cm})\end{array}$ & $\begin{array}{c}\text { Canopy } \\
\text { diameter } \\
(\mathrm{cm})\end{array}$ & $\begin{array}{c}\text { Root length } \\
(\mathrm{cm})\end{array}$ & $\begin{array}{c}\text { Root } \\
\text { volume (cc) }\end{array}$ & $\begin{array}{c}\text { No. of } \\
\text { truits/plant }\end{array}$ \\
\hline $\mathrm{S}_{1}$ & 305.88 & 1840.51 & 65.84 & 41.32 & 436.48 & 9.73 & 128.19 \\
$\mathrm{~S}_{2}$ & 398.15 & 2036.28 & 73.58 & 48.58 & 467.39 & 10.03 & 185.98 \\
$\mathrm{~S}_{2}$ & 368.07 & 1951.22 & 68,53 & 45.26 & 523.62 & 11,00 & 176.57 \\
\hline $\mathrm{LSD}(0.05)$ & 19.87 & 1.714 & 0.214 & 20.72 & 1.764 & 0.375 & 5.35 \\
$\mathrm{CV}(\%)$ & 7.35 & 4.47 & 9.10 & 8.89 & 13.17 & 8,83 & 13.68 \\
\hline
\end{tabular}

Table 2. Cont'd.

\begin{tabular}{l|c|c|c|c|c|c|c|c}
\hline Growth & $\begin{array}{c}\text { Fruit } \\
\text { length } \\
(\mathrm{mm})\end{array}$ & $\begin{array}{c}\text { fruit } \\
\text { diameter } \\
(\mathrm{mm})\end{array}$ & $\begin{array}{c}\text { Individual } \\
\text { fruit wt } \\
(\mathrm{mg})\end{array}$ & $\begin{array}{c}\text { Fruit } \\
\text { yield/ } \\
\text { plant (g) }\end{array}$ & $\begin{array}{c}\text { Leaf dry } \\
\text { wt (g) }\end{array}$ & $\begin{array}{c}\text { Stem dry } \\
\text { wt (g) }\end{array}$ & $\begin{array}{c}\text { Root dry } \\
\text { wt (g) }\end{array}$ & $\begin{array}{c}\text { Fruit dry } \\
\text { wt/plant } \\
\text { (g) }\end{array}$ \\
\hline $\mathrm{S}_{1}$ & 32.56 & 8.02 & 549.60 & 73.84 & 3.60 & 7.89 & 2.40 & 15.51 \\
$\mathrm{~S}_{2}$ & 27.25 & 6.93 & 377.15 & 72.17 & 4.02 & 9.02 & 2.60 & 14.04 \\
$\mathrm{~S}_{3}$ & 30.86 & 7.62 & 443.68 & 79.40 & 3.67 & 8.24 & 2.84 & 17.74 \\
\hline $\mathrm{I}, \mathrm{SD}(0.05)$ & 1.714 & 0.214 & 20.72 & 1.764 & 0.117 & 0.275 & 0.086 & 0.555 \\
$\mathrm{CV}(\%)$ & 11.80 & 10.81 & 13.32 & 12.98 & 9.90 & 7.56 & 11.78 & 11.29 \\
\hline
\end{tabular}

${ }^{*} \mathrm{~S}_{1}=$ Vegetative stage, $\mathrm{S}_{2}=$ Flowering stage and $\mathrm{S}_{3}=$ Fruiting stage.

Effects of six water treatments applied in this experiment on different physiomorphological and fruiting characters are shown in the Table 3. In this Table, the water treatments like $\mathrm{W}_{1}, \mathrm{~W}_{2}, \mathrm{~W}_{5}$, and $\mathrm{W}_{0}$ showed their stress effects either due to excess or deficit moisture on the parameters studied. On the other hand, the treatments like $\mathrm{W}_{3}$ and $\mathrm{W}_{4}$ are expressed as optimum and semi optimum levels, respectively, of moisture for chilli plants. The values for the parameters varied significantly between optimum and stress water treatments. The water treatment of $\mathrm{W}_{3}$ produced the highest values for the parameters and was followed by that of $\mathrm{W}_{4}$ and lower values were produced by the stress treatments like $\mathrm{W}_{1}$ and $\mathrm{W}_{2}$ (excess water) and $\mathrm{W}_{5}$ and $\mathrm{W}_{0}$ (Table 3 ).

The number of leaves and leaf area per plant has been found to be small under deficit and excess moisture treatments of $\mathrm{W}_{5}, \mathrm{~W}_{0}, \mathrm{~W}_{1}$, and $\mathrm{W}_{2}$ (Table 3). Horton et al. (1982) reported fewer leaves per plant with the drier treathent. Lower leaf area with drier treatment was also observed by Beese et al. (1982) in their experiment conducted on chilli varieties. So, the present findings on the number of leaves and leaf area are in agreement with those findings. The plant height, root length, root volume, leaf dry weight, stem dry weight and root dry weight were also affected by the water stress treatments (Table 3). 
Techawongstein et al. (1992) observed suppression of plant height due to water stress in chilli. So, the plant height reduction in the study is supported by the above cited reference. Beese et al. (1982) reported reduction in final yields of above and below ground plant parts in chilli due to moisture stress effect. Many research workers found lower values of different plant parts as a result of water stress (Ayob, 1986; Beese and Moshrefi. 1985; Hedge, 1989; Smittle et al, 1994). The excess water caused lower yield in chilli as reported by Wankhede and Morey (1984). The present findings due to deficit and excess moisture treatment on most of the growth parameters reflect the reports of the above mentioned authors.

Table 3. Effect of water treatment on the physio-morphological characters of two cultivars (accessions) of chilli.

\begin{tabular}{l|c|c|c|c|c|c|c}
\hline $\begin{array}{c}\text { Water } \\
\text { treatments* }\end{array}$ & $\begin{array}{c}\text { No. of } \\
\text { leaves/ } \\
\text { plant }\end{array}$ & $\begin{array}{c}\text { Leaf area/ } \\
\text { plant }\left(\mathrm{cm}^{2}\right)\end{array}$ & $\begin{array}{c}\text { Plant } \\
\text { height } \\
(\mathrm{cm})\end{array}$ & $\begin{array}{c}\text { Canopy } \\
\text { diameter } \\
(\mathrm{cm})\end{array}$ & $\begin{array}{c}\text { Root } \\
\text { length } \\
(\mathrm{cm})\end{array}$ & $\begin{array}{c}\text { Root } \\
\text { volume } \\
(\mathrm{cc})\end{array}$ & $\begin{array}{c}\text { No. of } \\
\text { truits/ plant }\end{array}$ \\
\hline $\mathrm{W}_{1}$ & 292.43 & 1662.62 & 96.14 & 41.16 & 464.27 & 10.09 & 138.83 \\
$\mathrm{~W}_{2}$ & 378.72 & 1837.38 & 70.68 & 45.51 & 454.57 & 9.79 & 159.73 \\
$\mathrm{~W}_{3}$ & 451.02 & 2862.86 & 73.49 & 54.42 & 508.14 & 11.23 & 206.10 \\
$\mathrm{~W}_{4}$ & 408.67 & 1957.25 & 71.72 & 48.50 & 482.92 & 10.71 & 193.44 \\
$\mathrm{~W}_{5}$ & 316.15 & 1737.06 & 68.16 & 42.05 & 489.69 & 10.25 & 149.14 \\
$\mathrm{~W}_{0}$ & 297.21 & 1598.85 & 62.70 & 38.68 & 455.37 & 9.46 & 134.23 \\
\hline LSD(0.05) & 44.11 & 50.94 & 5.42 & 2.800 & 35.99 & 0.334 & 8.64 \\
CV (\%) & 7.35 & 4.47 & 9.10 & 8.89 & 13.17 & 8.83 & 13.68 \\
\hline
\end{tabular}

Table 3. Cont'd.

\begin{tabular}{l|cc|c|c|c|c|c|c}
\hline Water & $\begin{array}{c}\text { Fruit } \\
\text { length } \\
\text { treatments* }\end{array}$ & $\begin{array}{c}\text { fruit } \\
\text { diameter } \\
(\mathrm{mm})\end{array}$ & $\begin{array}{c}\text { Individual } \\
\text { fruit wt } \\
(\mathrm{mg})\end{array}$ & $\begin{array}{c}\text { Fruit } \\
\text { yield/ } \\
\text { plant (g) }\end{array}$ & $\begin{array}{c}\text { Leaf dry } \\
\text { wt (g) }\end{array}$ & $\begin{array}{c}\text { Stem dry } \\
\text { wt (g) }\end{array}$ & $\begin{array}{c}\text { Root dry } \\
\text { wt (g) }\end{array}$ & $\begin{array}{c}\text { Fruit dry } \\
\text { wt/ plant } \\
\text { (g) }\end{array}$ \\
\hline $\mathrm{W}_{1}$ & 25.58 & 7.05 & 422.51 & 57.34 & 3.21 & 7.88 & 2.52 & 11.01 \\
$\mathrm{~W}_{2}$ & 28.57 & 7.45 & 422.21 & 66.12 & 3.88 & 8.47 & 2.09 & 11.91 \\
$\mathrm{~W}_{3}$ & 39.59 & 8.72 & 574.67 & 116.19 & 4.64 & 9.22 & 3.40 & 25.87 \\
$\mathrm{~W}_{4}$ & 35.73 & 7.83 & 480.61 & 90.91 & 4.16 & 8.91 & 3.18 & 18.93 \\
$\mathrm{~W}_{5}$ & 26.89 & 7.30 & 432.94 & 63.30 & 3.47 & 8.17 & 2.79 & 14.13 \\
$\mathrm{~W}_{0}$ & 24.99 & 6.79 & 407.92 & 56.97 & 3.22 & 7.59 & 1.71 & 12.72 \\
\hline $\mathrm{L}, \mathrm{SD}(0.05)$ & 2.471 & 0.736 & 33.40 & 2.525 & 0.277 & 0.531 & 0.305 & 0.714 \\
$\mathrm{CV}(\%)$ & 11.8 & 10.81 & 13.32 & 12.98 & 9.90 & 7.56 & 11.78 & 11.29 \\
\hline
\end{tabular}

$* \mathrm{~W}_{1}=$ watering once everyday, $\mathrm{W}_{2}=$ watering twice everyday, $\mathrm{W}_{3}=$ watering at 4 days interval, $\mathrm{W}_{4}=$ watering at 8 days interval, $\mathrm{W}_{5}=$ watering at 16 days interval and $\mathrm{W}_{0}=$ no watering (control). 
Table 4. Combined effect of growth stage $x$ water treatment $x$ cultivar on the physio-morphological characters of chilli.

\begin{tabular}{|c|c|c|c|c|c|c|c|}
\hline Treatments * & $\begin{array}{c}\text { No. of } \\
\text { leaves/ } \\
\text { plant }\end{array}$ & $\begin{array}{l}\text { Leaf area/ } \\
\text { plant }\left(\mathrm{cm}^{2}\right)\end{array}$ & $\begin{array}{c}\text { Plant } \\
\text { height } \\
(\mathrm{cm})\end{array}$ & $\begin{array}{c}\text { Canopy } \\
\text { diameter } \\
(\mathrm{cm})\end{array}$ & $\begin{array}{l}\text { Root } \\
\text { length } \\
(\mathrm{cm})\end{array}$ & $\begin{array}{c}\text { Root } \\
\text { volume } \\
\text { (cc) }\end{array}$ & $\begin{array}{l}\text { No. of } \\
\text { fruits/ } \\
\text { plant }\end{array}$ \\
\hline $\mathrm{S}_{1} \mathrm{~W}_{1} \mathrm{~V}_{1}$ & 266.20 & 1791.80 & 75.63 & 42.80 & 488.63 & 10.33 & 128.96 \\
\hline $\mathrm{S}_{1} \mathrm{~W}_{1} \mathrm{~V}_{2}$ & 212.20 & 1340.20 & 58.53 & 26.93 & 374.17 & 8.43 & 92.64 \\
\hline $\mathrm{S}_{1} \mathrm{~W}_{2} \mathrm{~V}_{1}$ & 334.43 & 1962.87 & 69.73 & 48.10 & 482.40 & 10.00 & 144.16 \\
\hline $\mathrm{S}_{1} \mathrm{~W}_{2} \mathrm{~V}_{2}$ & 298.63 & 1496.63 & 63.30 & 32.90 & 355.70 & 8.17 & 100.16 \\
\hline $\mathrm{S}_{1} \mathrm{~W}_{3} \mathrm{~V}_{1}$ & 430.07 & 2825.93 & 72.90 & 60.27 & 496.43 & 11.93 & 180.96 \\
\hline $\mathrm{S}_{1} \mathrm{~W}_{3} \mathrm{~V}_{2}$ & 389.60 & 2579.33 & 63.06 & 41.53 & 418.23 & 9.94 & 120.79 \\
\hline $\mathrm{S}_{1} \mathrm{~W}_{4} \mathrm{~V}_{1}$ & 350.90 & 2004.07 & 71.20 & $51 \$ ?$ & 458.67 & 11.43 & 160.18 \\
\hline $\mathrm{S}_{1} \mathrm{~W}_{4} \mathrm{~V}_{2}$ & 277.90 & 1676.77 & 62.23 & 37.10 & 372.60 & 9.23 & 104.04 \\
\hline $\mathrm{S}_{1} \mathrm{~W}_{5} \mathrm{~V}_{1}$ & 296.93 & 1849.30 & 69.40 & 45.50 & 489.53 & 11.07 & 138.91 \\
\hline $\mathrm{S}_{1} \mathrm{~W}_{5} \mathrm{~V}_{2}$ & 232.00 & 1420.13 & 57.77 & 30.00 & 487.79 & 8.80 & 98.88 \\
\hline $\mathrm{S}_{1} \mathrm{~W}_{0} \mathrm{~V}_{1}$ & 329.34 & 1739.26 & 67.68 & 47.99 & 457.53 & 9.50 & 153.13 \\
\hline $\mathrm{S}_{1} \mathrm{~W}_{0} \mathrm{~V}_{2}$ & 252.34 & 1399.79 & 58.65 & 30.87 & 359.03 & 7.87 & 11.41 \\
\hline $\mathrm{S}_{2} \mathrm{~W}_{1} \mathrm{~V}_{1}$ & 377.40 & 2000.30 & 78.40 & 53.70 & 504.90 & 10.90 & 182.40 \\
\hline $\mathrm{S}_{2} \mathrm{~W}_{1} \mathrm{~V}_{2}$ & 287.40 & 1495.40 & 66.20 & 39.50 & 370.50 & 8.93 & 130.40 \\
\hline $\mathrm{S}_{2} \mathrm{~W}_{2} \mathrm{~V}_{1}$ & 480.27 & 2195.07 & 80.37 & 57.13 & 504.87 & 10.57 & 206.72 \\
\hline $\mathrm{S}_{2} \mathrm{~W}_{2} \mathrm{~V}_{2}$ & 389.90 & 1681.70 & 72.27 & 43.73 & 386.23 & 8.73 & 162.56 \\
\hline $\mathrm{S}_{2} \mathrm{~W}_{3} \mathrm{~V}_{1}$ & 529.57 & 3417.10 & 86.57 & 67.57 & 595.45 & 12.41 & 282.72 \\
\hline $\mathrm{S}_{2} \mathrm{~W}_{3} \mathrm{~V}_{2}$ & 453.73 & 2558.57 & 71.87 & 48.17 & 408.02 & 9.44 & 197.12 \\
\hline $\mathrm{S}_{2} \mathrm{~W}_{4} \mathrm{~V}_{1}$ & 494.70 & 2255.03 & 85.17 & 59.57 & 553.63 & 11.57 & 262.24 \\
\hline $\mathrm{S}_{2} \mathrm{~W}_{4} \mathrm{~V}_{2}$ & 426.33 & 1875.23 & 70.60 & 44.27 & 424.77 & 9.37 & I96.80 \\
\hline $\mathrm{S}_{2} \mathrm{~W}_{5} \mathrm{~V}_{1}$ & 404.63 & 2077.87 & 77.10 & 53,90 & 517.50 & 10.93 & 192.00 \\
\hline $\mathrm{S}_{2} \mathrm{~W}_{5} \mathrm{~V}_{2}$ & 323.87 & 1591.50 & 69.90 & 39.00 & 406.83 & 9.10 & 147.80 \\
\hline $\mathrm{S}_{2} \mathrm{~W}_{0} \mathrm{~V}_{1}$ & 345.03 & 1976.43 & 65.93 & 49.49 & 49.53 & 10.07 & 167.00 \\
\hline $\mathrm{S}_{2} \mathrm{~W}_{0} \mathrm{~V}_{2}$ & 264.98 & 1311.21 & 58.64 & 29.96 & 438.43 & 8.40 & 103.98 \\
\hline $\mathrm{S}_{3} \mathrm{~W}_{1} \mathrm{~V}_{1}$ & 344.60 & 1913.93 & 74.47 & 50.57 & 561.80 & 11.64 & 173.28 \\
\hline $\mathrm{S}_{3} \mathrm{~W}_{1} \mathrm{~V}_{2}$ & 266.80 & 1434.07 & 61.60 & 33.43 & 488.60 & 10.29 & 125.28 \\
\hline $\mathrm{S}_{3} \mathrm{~W}_{2} \mathrm{~V}_{1}$ & 435.73 & 2090.30 & 73.17 & 52.60 & 551.76 & 11.60 & 194.56 \\
\hline $\mathrm{S}_{3} \mathrm{~W}_{2} \mathrm{~V}_{2}$ & 333.33 & 1597.70 & 65.27 & 38.60 & 446.56 & 9.67 & 150,24 \\
\hline $\mathrm{S}_{3} \mathrm{~W}_{3} \mathrm{~V}_{1}$ & 510.43 & 3033.73 & 77.23 & 64.83 & 670.90 & 13.07 & 265.92 \\
\hline $\mathrm{S}_{3} \mathrm{~W}_{3} \mathrm{~V}_{2}$ & 392.70 & 2762.50 & 69.30 & 44.17 & 459.83 & 10.60 & 189.12 \\
\hline $\mathrm{S}_{3} \mathrm{~W}_{4} \mathrm{~V}_{1}$ & 486.10 & 2137.83 & 75.87 & 57.17 & 587.93 & 12.53 & 250.24 \\
\hline $\mathrm{S}_{3} \mathrm{~W}_{4} \mathrm{~V}_{2}$ & 416.10 & 1794.57 & 65.30 & 41.03 & 499.90 & 10.10 & 187.12 \\
\hline $\mathrm{S}_{3} \mathrm{~W}_{5} \mathrm{~V}_{1}$ & 349.63 & 1965.53 & 71.43 & 39.53 & 564.20 & 11.87 & 181.76 \\
\hline $\mathrm{S}_{3} \mathrm{~W}_{5} \mathrm{~V}_{2}$ & 289.84 & 1518.00 & 63.37 & 34.37 & 472.30 & 9.73 & 134.52 \\
\hline $\mathrm{S}_{3} \mathrm{~W}_{0} \mathrm{~V}_{1}$ & 313.65 & 1705.58 & 66.87 & 42.52 & 529.53 & 11.43 & 141.25 \\
\hline $\mathrm{S}_{3} \mathrm{~W}_{0} \mathrm{~V}_{2}$ & 277.92 & 1460.84 & 58.45 & 34.26 & 450.13 & 9.47 & 124.58 \\
\hline $\operatorname{LSD}(0.05)$ & 42.02 & 138.82 & 10.09 & 6.412 & 100.31 & 1.448 & 35.80 \\
\hline CV (\%) & 7.35 & 4.47 & 9.10 & 8.89 & 13.17 & 8.83 & 13.68 \\
\hline
\end{tabular}


Table 4. Cont'd.

\begin{tabular}{|c|c|c|c|c|c|c|c|c|}
\hline Treatments* & $\begin{array}{l}\text { Fruit } \\
\text { length } \\
(\mathrm{mm})\end{array}$ & $\begin{array}{c}\text { Fruit } \\
\text { diameter } \\
(\mathrm{mm})\end{array}$ & $\begin{array}{l}\text { Individual } \\
\text { fruit } \\
\text { weight } \\
\text { (mg) } \\
\end{array}$ & $\begin{array}{c}\text { Fruit } \\
\text { yield/ } \\
\text { plant (g) }\end{array}$ & $\begin{array}{l}\text { Leaf dry } \\
\text { wt (mg) }\end{array}$ & $\begin{array}{l}\text { Stem } \\
\text { dry wt } \\
\text { (mg) }\end{array}$ & $\begin{array}{l}\text { Root } \\
\text { dry wt } \\
\text { (mg) }\end{array}$ & $\begin{array}{c}\text { Fruit } \\
\text { dry wt/ } \\
\text { plant } \\
\text { (g) }\end{array}$ \\
\hline $\mathrm{S}_{1} \mathrm{~W}_{1} \mathrm{~V}_{1}$ & 32.20 & 8.17 & 526.50 & 67.88 & 3.70 & 8.27 & 2.80 & 13.28 \\
\hline $\mathrm{S}_{1} \mathrm{~W}_{1} \mathrm{~V}_{2}$ & 24.83 & 7.13 & 493.43 & 44.96 & 2.47 & 6.47 & 1.70 & 8.80 \\
\hline $\mathrm{S}_{1} \mathrm{~W}_{2} \mathrm{~V}_{1}$ & 37.80 & 8.37 & 557.83 & 80.40 & 4.13 & 9.23 & 2.53 & 13.76 \\
\hline $\mathrm{S}_{1} \mathrm{~W}_{2} \mathrm{~V}_{2}$ & 27.77 & 7.50 & 495.37 & 49.44 & 3.17 & 6.53 & 1.07 & 9.76 \\
\hline $\mathrm{S}_{1} \mathrm{~W}_{3} \mathrm{~V}_{1}$ & 48.07 & 11.17 & 813.63 & 147.22 & 4.83 & 9.37 & 4.03 & 27.84 \\
\hline $\mathrm{S}_{1} \mathrm{~W}_{3} \mathrm{~V}_{2}$ & 38.37 & 7.87 & 619.93 & 74.88 & 3.77 & 7.83 & 2.68 & 21.60 \\
\hline $\mathrm{S}_{1} \mathrm{~W}_{4} \mathrm{~V}_{1}$ & 44.00 & 8.60 & 628.60 & 100.68 & 4.53 & 9.07 & 3.53 & 22.56 \\
\hline $\mathrm{S}_{1} \mathrm{~W}_{4} \mathrm{~V}_{2}$ & 32.57 & 7.87 & 604.77 & 78.24 & 3.47 & 7.57 & 2.33 & 14.72 \\
\hline $\mathrm{S}_{1} \mathrm{~W}_{5} \mathrm{~V}_{1}$ & 32.93 & 8.47 & 577.70 & 80.20 & 3.87 & 8.28 & 3.03 & 16.48 \\
\hline $\mathrm{S}_{1} \mathrm{~W}_{5} \mathrm{~V}_{2}$ & 24.90 & 7.50 & 495.70 & 48.96 & 2.57 & 7.03 & 2.00 & 12.00 \\
\hline $\mathrm{S}_{1} \mathrm{~W}_{0} \mathrm{~V}_{1}$ & 26.95 & 7.04 & 393.90 & 67.62 & 4.12 & 8.13 & 2.07 & 13.65 \\
\hline $\mathrm{S}_{1} \mathrm{~W}_{0} \mathrm{~V}_{2}$ & 20.36 & 6.54 & 387.82 & 45.68 & 2.56 & 6.99 & 1.07 & 11.64 \\
\hline $\mathrm{S}_{2} \mathrm{~W}_{1} \mathrm{~V}_{1}$ & 24.20 & 6.80 & 351.00 & 64.00 & 4.00 & 9.37 & 3.03 & 11.84 \\
\hline $\mathrm{S}_{2} \mathrm{~W}_{1} \mathrm{~V}_{2}$ & 21.80 & 5.70 & 334.30 & 43.68 & 2.67 & 7.87 & 1.93 & 8.16 \\
\hline $\mathrm{S}_{2} \mathrm{~W}_{2} \mathrm{~V}_{1}$ & 26.13 & 7.67 & 370.93 & 76.64 & 4.80 & 10.03 & 2.83 & 12.80 \\
\hline $\mathrm{S}_{2} \mathrm{~W}_{2} \mathrm{~V}_{2}$ & 21.13 & 6.10 & 295.23 & 47.96 & 3.57 & 8.50 & 1.27 & 8.16 \\
\hline $\mathrm{S}_{2} \mathrm{~W}_{3} \mathrm{~V}_{1}$ & 40.57 & & 491.20 & 138.88 & 5.73 & 10.90 & 4.30 & 24.96 \\
\hline $\mathrm{S}_{2} \mathrm{~W}_{3} \mathrm{~V}_{2}$ & 29.53 & 6.83 & 440.90 & 86.88 & 4.43 & 9.20 & 2.51 & 18.88 \\
\hline $\mathrm{S}_{2} \mathrm{~W}_{4} \mathrm{~V}_{1}$ & 37.67 & 8.07 & 360.13 & 94.24 & 5.13 & 10.60 & 3.90 & 20.64 \\
\hline $\mathrm{S}_{2} \mathrm{~W}_{4} \mathrm{~V}_{2}$ & 27.87 & 6.90 & 405.13 & 79.72 & 4.00 & 8.83 & 2.53 & 13.60 \\
\hline $\mathrm{S}_{2} \mathrm{~W}_{5} \mathrm{~V}_{1}$ & 27.00 & 6.97 & 359.40 & 69.08 & 4.63 & 9.67 & 3.43 & 14.08 \\
\hline $\mathrm{S}_{2} \mathrm{~W}_{5} \mathrm{~V}_{2}$ & 20.40 & 5.77 & 341.83 & 50.56 & 2.93 & 8.17 & 2.23 & 9.76 \\
\hline $\mathrm{S}_{2} \mathrm{~W}_{0} \mathrm{~V}_{1}$ & 30.74 & 7.49 & 423.89 & 68.29 & 3.76 & 7.98 & 2.17 & 15.70 \\
\hline $\mathrm{S}_{2} \mathrm{~W}_{0} \mathrm{~V}_{2}$ & 19.99 & 6.00 & 351.90 & 46.10 & 2.56 & 7.10 & 1.10 & 9.91 \\
\hline $\mathrm{S}_{3} \mathrm{~W}_{1} \mathrm{~V}_{1}$ & 28.57 & 7.73 & 405.00 & 70.08 & 3.77 & 8.53 & 3.47 & 14.08 \\
\hline $\mathrm{S}_{3} \mathrm{~W}_{1} \mathrm{~V}_{2}$ & 21.90 & 6.77 & 424.83 & 63.44 & 2.63 & 6.80 & 2.17 & 9.92 \\
\hline $\mathrm{S}_{3} \mathrm{~W}_{2} \mathrm{~V}_{1}$ & 34.07 & 8.00 & 436.93 & 85.00 & 4.37 & 8.90 & 3.23 & 15.04 \\
\hline $\mathrm{S}_{3} \mathrm{~W}_{2} \mathrm{~V}_{2}$ & 24.50 & 7.07 & 376.97 & 57.28 & 3.27 & 7.60 & 1.63 & 11.92 \\
\hline $\mathrm{S}_{3} \mathrm{~W}_{3} \mathrm{~V}_{1}$ & 46.07 & 10.13 & 579.53 & 154.08 & 5.20 & 9.80 & 4.20 & 34.56 \\
\hline $\mathrm{S}_{3} \mathrm{~W}_{3} \mathrm{~V}_{2}$ & 34.93 & 7.47 & 502.83 & 95.20 & 3.90 & 8.23 & 2.70 & 27.36 \\
\hline $\mathrm{S}_{3} \mathrm{~W}_{4} \mathrm{~V}_{1}$ & 40.73 & 8.13 & 424.47 & 106.20 & 4.57 & 9.47 & 3.99 & 4.64 \\
\hline $\mathrm{S}_{3} \mathrm{~W}_{4} \mathrm{~V}_{2}$ & 31.57 & 7.40 & 460.57 & 86.40 & 3.27 & 7.90 & 2.80 & 17.44 \\
\hline $\mathrm{S}_{3} \mathrm{~W}_{5} \mathrm{~V}_{1}$ & 32.60 & 8.10 & 420.73 & 76.44 & 4.17 & 8.63 & 3.67 & 18.40 \\
\hline $\mathrm{S}_{3} \mathrm{~W}_{5} \mathrm{~V}_{2}$ & 23.50 & 7.00 & 402.30 & 54.56 & 2.63 & 7.30 & 2.37 & 14.08 \\
\hline $\mathrm{S}_{3} \mathrm{~W}_{0} \mathrm{~V}_{1}$ & 28.92 & 7.35 & 452.42 & 67.23 & 2.88 & 8.55 & 2.47 & 14.70 \\
\hline $\mathrm{S}_{3} \mathrm{~W}_{0} \mathrm{~V}_{2}$ & 22.95 & 6.30 & 437.58 & 46.88 & 2.43 & 6.82 & 1.37 & 10.71 \\
\hline $\mathrm{LSD}(0.05)$ & 5.706 & 1.30 & 73.17 & 7.992 & 0.597 & 1.013 & 0.49 & 2.847 \\
\hline CV (\%) & 11.80 & 10.8 & 13.32 & 13.98 & 9.90 & 7.56 & 11.7 & 11.29 \\
\hline
\end{tabular}

$* \mathrm{~S}_{1}=$ Vegetative stage, $\mathrm{S}_{2}=$ Flowering stage and $\mathrm{S}_{3}=$ Fruiting stage.

$\mathrm{W}_{1}=$ watering once everyday, $\mathrm{W}_{2}=$ watering twice everyday, $\mathrm{W}_{3}=$ watering at 4 days interval, $\mathrm{W}_{4}=$ watering at 8 days interval, $\mathrm{W}_{5}=$ watering at 16 days interval and $\mathrm{W}_{\mathrm{o}}=$ no watering (control). $\mathrm{V}_{1}=\mathrm{C} 0277$ and $\mathrm{V}_{2}=\mathrm{C} 0272$. 
The number of fruits per plant, fruit length and diameter, individual fruit weight, fruit yield per plant and fruit dry weight per plant were affected by the water stress of either deficit or excess conditions (Table 3). Techawongstein et al. (1992) from their experiment reported that in chilli, the number of fruits per plant, fruit length and diameter and individual fruit weight had been found to be less in water stressed plants than those in optimum level of water. Hedge (1989) observed adverse effect of both excess and deficit soil moisture on the fruit yield of chilli. Lower fruit yield in chilli was also reported by Ayob (1986) with the excess and deficit soil moisture. The plants had to maintain their moisture status within a certain range for their physiological activities. The growth and yield of chilli showed declining trend with higher levels of irrigations (Sadykov and Mikhoet, 1981). So, the present response of Physio-morphological and yield contributing characters to the excess water treatments are in agreement with the previous works.

From Table 4, it is observed that the water treatments in combination with other two factors like chilli accessions and growth stages of the experiment gave the same trend as in the Table 3 in producing values for all the fifteen parameters studied.

In case of all the possible combinations of water treatments, accessions and growth stages, the accession $\mathrm{V}_{1}(\mathrm{C}-0277)$ produced higher values for all the parameters (Table 4). From this Table, it is also evident that the water treatments of $\mathrm{W}_{3}$ and $\mathrm{W}_{4}$ were the highest and second highest value producers, respectively and the excess water treatments of $\mathrm{W}_{1}$ and $\mathrm{W}_{2}$ and deficit water treatments of $\mathrm{W}_{5}$ and $\mathrm{W}_{0}$ are the lower value producers for all the parameters studied. In the vegetative stage, the number of fruits per plant showed sensitivity to the excess moisture conditions. The leaf, stem and root dry weight subjected to stress treatments at the vegetative stage is lower on average than that of the mature stages (Table 2). Techawongstein et al. (1992) observed similar result with the same parameters. The stress developed during the treatment period markedly suppressed the vegetative growth and the plant became stunted. Although the yield decreased significantly by stress at all stages, number of fruits was more affected than the individual fruit weight. When the stress was given at later stages, the reduction in yield has not been found to be that serious (Table 4). Techawongstein et al. (1992) forwarded similar observation from their experiment on chilli. According to them when the plants were exposed to deficit water at the vegetative stage, there was decrease in the fruit number, while treated at the matured stage there was decrease in the fruit weight. The reduction in number of fruits in the vegetative stage had been found to occur due to poor 
flower bud formation and development of fruit. This reduction in number of fruits was, however, compensated by the increase in the fruit weight to some extent. Decrease in fruit weight in the matured stages treatment might be due to

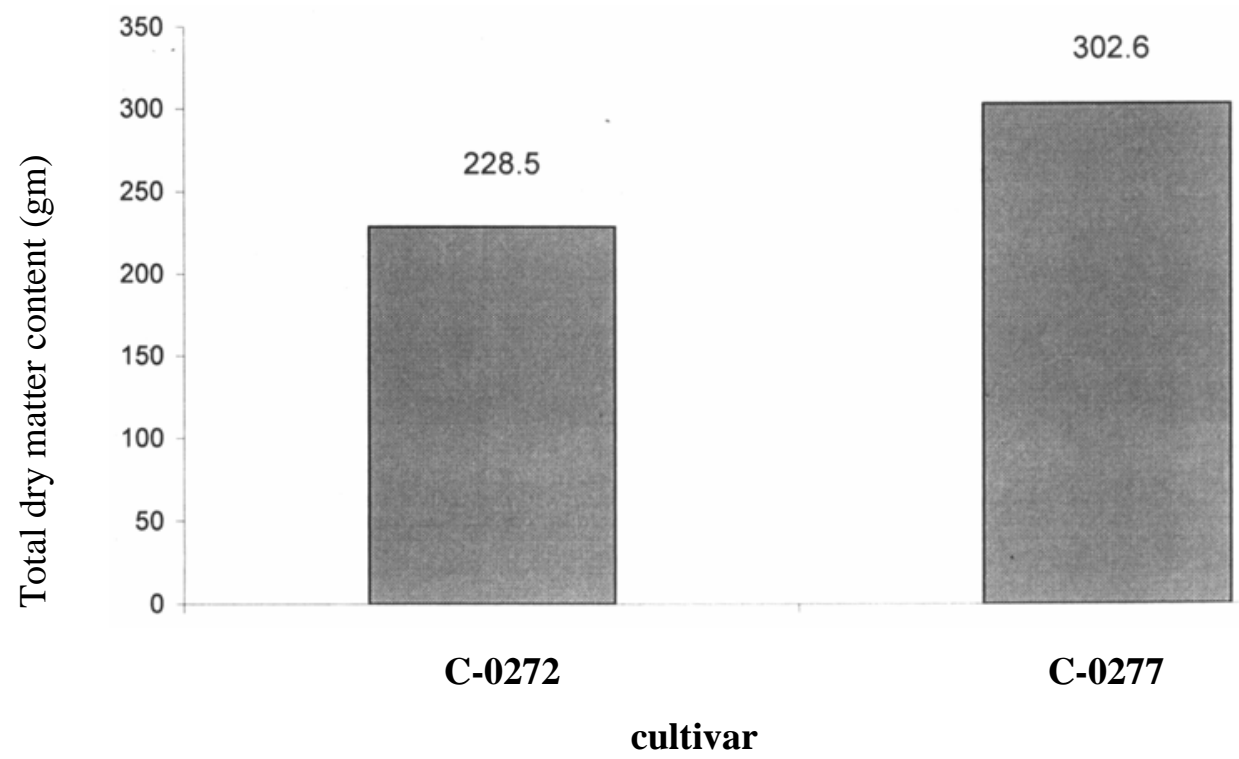

Fig. 1. Total dry matter content of two selected chilli cultivars treated under SxWxV treatment combinations in the field.

the competition between vegetative and reproductive organs which ultimately reflected on the yield. The present findings of the effect of stress treatment at the vegetative and matured stages are supported by the above cited statements. Better performance of the chilli accession, C-O277 with all the water treatments including stress treatments imposed at all the three growth stages proved its tolerances to water stress. On the other hand, the accession C-0272 gave the lowest values in case of all test parameters in combination with all the water treatments including stress ones proves its susceptibility to water stress. It was revealed that the accessions C-O27 and C-0272 produced higher (302.6 g) and lower (228.5 g) content of total dry matter, respectively, when treated by the water treatments including stress ones (Fig. 1).

\section{Conclusion}

The chilli accessions C-0277 and C-0272 could be identified from this field experiment as water stress tolerant and susceptible chilli for cultivation in Bangladesh. 


\section{References}

Ayob, K. 1986. Effect of available soil moisture on the yield of chilli (Capsicum annuum). Technology Sayur Sayuran 2: 57-59.

Beese, F. and N. Moshrefi. 1985. Physiological reaction of chilli pepper to water and salt stress. Dirppertrickle Irrigation in Action 2: 646-651.

Beese, F., R. Horton and P.J. Wierenga. 1982. Growth and yield response of chilli pepper to trickle irrigation. Agron. J. 74: 556-561.

Hegde. D. M. 1989. Effect of soil moisture and nitrogen on plant water relations, mineral composition and productivity of bell pepper (Capsicum annuum). Indian J. Agron. 34 : 30-34.

Horton, R., F. Beese and P.J. Wierenga. 1982. Physiological response of chilli pepper to trickle irrigation. Agron. J. 74: 55 1-555.

Sadykov, I. M. and Zh. Yu. Mikhoet. 1981. Vegetable Crops in India. Naya Prokash, Calcutta. pp. 320-329.

Smittle, D.A., W.L. Dickens and J.R. Stansell. 1994. Irrigation regimes affect yield and water use by bell pepper. J. Amer. Soc. Hort. Sci. 119(5): 936-939.

Techawongstein, S., E. Nawata and S. Shigenaga. 1992. Effect of water stress at various stages of plant development on growth and yield of chilli pepper. Japan. J. Trop. Agr. 36(1): 5 1-57.

Wankhede, B. N. and D.K. Morey. 1984. Effect of levels of irrigation, nitrogen and potash on the growth and yield of chilli. Punjabrao Krishi Vidyapeeth. Res. J. 8(2): 22-25. 